\title{
MODIFIED SECOND ORDER SLOPE ROTATABLE DESIGNS USING SYMMETRICAL UNEQUAL BLOCK ARRANGEMENTS WITH TWO UNEQUAL BLOCK SIZES
}

B. Re. Victorbabu*

\section{ABSTRACT}

In this paper, a new method of construction of modified second order slope rotatable designs using symmetrical unequal block arrangements with two unequal sizes are constructed.

\section{Introduction}

Box and Hunter (1957) introduced rotatable designs for the exploration of response surfaces. Das and Narasimham (1962) constructed rotatable designs through balanced incomplete block designs (BIBD). Das et al. (1999) constructed response surface designs, symmetrical and asymmetrical, rotatable and modified.

The study of rotatable designs mainly emphasized on the estimation of absolute response. Estimation of differences in response at two different points in the factor space will often be of great importance. If differences at two points close together,

- Department of Statistics, Acharya Nagariuna University, Guntur - 522510 (A.P.), India 
estimation of local slope (rate of change) of the response is of interest. Estimation of slopes occurs frequently in practical situations. For instance, there are cases in which we want to estimate rate of reaction in chemical experiment, rate of change in the yield of a crop to various fertilizer doses, rate of disintegration of radioactive material in an animal etc., Hader and Park (1978) introduced slope rotatable central composite designs. Park (1987) introduced a class of multifactor designs for estimating the slope of response surfaces. Victorbabu and Narasimham (1991) studied general second order slope rotatable designs (SOSRD) and constructed SOSRD using BIBD. Jang and Park (1993) studied a measure and graphical method for evaluating slope rotatability in response surface designs. Victorbabu (2002a) suggested a note on the construction of four and six level SOSRD. Victorbabu (2002b) constructed SOSRD using symmetrical unequal block arrangements with two unequal sizes. Victorbabu (2003) studied SOSRD using incomplete block designs. Victorbabu (2005) studied modified SOSRD using pairwise balanced designs.

\section{Conditions for Modified SOSRD}

A second order response surface design $D=\left(\left(x_{i j}\right)\right)$ for fitting,

$$
Y_{v}=b_{0}+\sum_{i=1}^{v} b_{i} x_{i v}+\sum_{i=1}^{v} b_{i i} x_{i v}^{2}+\sum \sum b_{i j} x_{i v} x_{i v}+e_{v}
$$

where $x_{i v}$ denotes the level of the $i^{\text {it }}$ factoriki $\left.=1,2, \ldots, v\right)$ in the $u^{\text {th }}$ run $(u=1,2, \ldots, N)$ of the experiment, $e_{v}$ 's are uncorrelated random errors with mean zero and variance $\sigma^{2}$.

SOSRD: A second order response surface design $D$ is said to be a SOSRD, if the variance of the estimate of first order partial derivative $\left(\partial \hat{Y}_{v} / \partial x_{i}\right)$ with respect to each of independent variables $\left(x_{i}\right)$ is only a function of the distance $\left(d^{2}=\sum_{i} x_{i v}^{2}\right)$ of the point $\left(x_{1 v^{\prime}}, x_{2 v^{\prime}}, \ldots, x_{v v}\right)$ from the origin (centre) of the design. Such a spherical variance function for estimation of slopes in the second order response surface is achieved if the design points satisfy the following conditions (cf. Hader and Park (1978), Victorbabu and Narasimham (1991)).

$$
\begin{aligned}
& \sum_{v=1}^{N} \prod_{i=1}^{v} x_{i v}^{\alpha_{i}}=0 \text { if any } \alpha_{i} \text { is odd, for } \sum \alpha_{i} \leq 4 \\
& \sum_{v=1}^{N} x_{i v}^{2}=\text { constant }=N \lambda_{2}
\end{aligned}
$$


(ii) $\quad \sum_{v=1}^{N} x_{i v}^{4}=$ constant $=C N \lambda_{4}$, for all i

$$
\begin{aligned}
& \sum_{v=1}^{N} x_{i v}^{2} x_{i v}^{2}=\text { constant }=N \lambda_{4}, \text { for } i \neq i \\
& (c+v-1) \lambda_{4}>v \lambda_{2}^{2} \\
& \lambda_{4}\left[v(5-c)-(c-3)^{2}\right]+\lambda_{2}^{2}[v(c-5)+4]=0
\end{aligned}
$$

where $c, \lambda_{2}$ and $\lambda_{4}$ are constants and the summation is over the design points.

The variances and co-variances of the estimated parameters are,

$$
\begin{aligned}
& V\left(\hat{b}_{0}\right)=\frac{\lambda_{4}(c+v-1) \sigma^{2}}{N\left[\lambda_{4}(c+v-1)-v \lambda_{2}^{2}\right]} \\
& V\left(\hat{b}_{i}\right)=\frac{\sigma^{2}}{N \lambda_{2}} \\
& V\left(\hat{b}_{i}\right)=\frac{\sigma^{2}}{N \lambda_{4}} \\
& V\left(\hat{b}_{i i}\right)=\frac{\sigma^{2}}{(c-1) N \lambda_{4}}\left[\frac{\lambda_{4}(c+v-2)-(v-1) \lambda_{2}^{2}}{\lambda_{4}(c+v-1)-v \lambda_{2}^{2}}\right] \\
& \operatorname{Cov}\left(\hat{b}_{0}, \hat{b}_{i i}\right)=\frac{-\lambda_{2} \sigma^{2}}{N\left[\lambda_{4}(c+v-1)-v \lambda_{2}^{2}\right]} \\
& \operatorname{Cov}\left(\hat{b}_{i i}, \hat{b}_{i j}\right)=\frac{\left(\lambda_{2}{ }^{2}-\lambda_{4}\right) \sigma^{2}}{(c-1) N \lambda_{4}\left[\lambda_{4}(c+v-1)-v \lambda_{2}^{2}\right]}
\end{aligned}
$$

and other co-variances are zero.

$$
V\left(\frac{\partial \hat{Y}}{\partial x_{i}}\right)=V\left(\hat{b}_{i}\right)+4 x_{i}^{2} V\left(\hat{b}_{i i}\right)+\sum_{i * i} x_{i}^{2} V\left(\hat{b}_{i j}\right)=\frac{1}{N}\left[\frac{\lambda_{4}+\lambda_{2} d^{2}}{\lambda_{2} \lambda_{4}}\right] \sigma^{2}
$$


The usual method of construction of SOSRD is to take combinations with unknown constants, associate a $2^{v}$ factorial combinations or a suitable fraction of it with factors each at +1 and -1 levels to make the level codes equidistant. All such combinations form a design. Generation of design points this way ensures satisfaction of all the conditions even though the design points contain unknown levels.

Alternatively by putting some restrictions indicating some relation among $\sum x_{i u}^{2}$, $\sum x_{i u}^{4}$ and $\sum x_{i v}^{2} x_{i v}^{2}$ some equations involving the unknowns are obtained and their solution gives the unknown levels. In SOSRD the restriction used is $V\left(b_{i}\right)=4 V\left(b_{i}\right)$ viz. equation (2.6). Other restrictions are also possible though, it seems, not yet exploited. We shall investigate the restriction $\left(\sum x_{i v}^{2}\right)^{2}=N \sum x_{i v}^{2} x_{i v}^{2}$ i.e., $\left(N \lambda_{2}\right)^{2}=N\left(N \lambda_{4}\right)$ to get modified SOSRD. By applying the new modification of Das et al. (1999) restriction in equation (2.6), we get $c=5$. Further,

$$
\begin{aligned}
& V\left(\hat{b}_{0}\right)=\frac{(v+4) \sigma^{2}}{4 N} \\
& V\left(\hat{b}_{i}\right)=\frac{\sigma^{2}}{N \sqrt{\lambda_{4}}} \\
& V\left(\hat{b}_{i j}\right)=\frac{\sigma^{2}}{N \lambda_{4}} \\
& V\left(\hat{b}_{i i}\right)=\frac{\sigma^{2}}{4 N \lambda_{4}} \\
& \operatorname{Cov}\left(\hat{b}_{0}, \hat{b}_{i i}\right)=\frac{-\sigma^{2}}{4 N \sqrt{\lambda_{4}}} \text { and other co-variances are zero. } \\
& V\left(\frac{\partial \hat{Y}}{\partial x_{i}}\right)=\left[\frac{\sqrt{\lambda_{4}}+d^{2}}{N \lambda_{4}}\right] \sigma^{2}:
\end{aligned}
$$




\section{Construction Of Modified SOSRD Using Symmetrical Unequal Block Arrangements With Two Unequal Block Sizes}

The method of construction of modified SOSRD using symmetrical unequal block arrangements (SUBA) with two unequal block sizes is given in theorem (3.1). Here we use the notations of Raghavarao (1971), pp. 291-292 and 298-300, Victorbabu and Narasimham (1991), pp. 2467-2472.

\section{SUBA with Two Unequal Block Sizes (c.f. Raghavarao, 1962)}

The arrangement of v-treatments in $b$ blocks where $b_{1}$ blocks of size $k_{1}$ and $b_{2}$ blocks of size $k_{2}$ is said to be a symmetrical unequal block arrangement with two unequal block sizes, if

(i) every treatment occurs $\frac{b_{i} k_{i}}{v_{n u}}$ blocks of size $k_{i}(i=1,2)$, and

(ii) every pair of first associate treatments occurs together in $u$ blocks of size $k_{1}$ and in $(\lambda-u)$ blocks of size $k_{2}$ while every pair of second associate treatments occurs together in $\lambda$ blocks of size.

From (i) each treatment occurs in $\left(\frac{b_{1} k_{1}}{v_{n v}}\right)+\left(\frac{b_{2} k_{2}}{v_{n v}}\right)=r$ blocks in all. $\left(v, b, r, k_{1}, k_{2}\right.$ $b_{1}, b_{2}, \lambda$ ) are known as the parameters of the SUBA with two unequal block sizes.

Let $\left(v, b, r, k_{1}, k_{2}, b_{1}, b_{2}, \lambda\right), k=\sup \left(k_{1}, k_{2}\right)$ and $b_{1}+b_{2}=b$ be a SUBA with two unequal block sizes. $2^{\text {th) }}$ denotes a resolution $V$ fractional factorial of $2^{k}$ in +1 levels, such that no interaction with less than five factors is confounded.

$\left[1-\left(v, b, r, k_{1}, k_{2}, b_{1}, b_{2}, \lambda\right)\right]$ denote the design points generated from the transpose of incidence matrix of SUBA with two unequal block sizes, []$-(v, b, r$, $\left.\left.k_{1}, k_{2}, b_{1}, b_{2}, \lambda\right)\right] 2^{\text {t(k) }}$ are the $b 2^{\text {t(k) }}$ design points generated from SUBA with two unequal block sizes by multiplication (c.f. Raghavarao (1971), pp. 298-300), (a, $0,0, \ldots, 0) 2^{\prime}$ denote the design points generated from $(a, 0,0 \ldots, 0)$ point set, and $U$ denotes combination of the design points generated from different sets of points. Repeat this set of additional design points say $n_{0}$ times when $r<5 \lambda$. Let $(a, a$, 
$\ldots, a) 2^{\prime(v)}$ denote the design points generated from $(a, a, \ldots, a)$ point set. Repeat this set of additional design points say $n_{a}$ times when $r>5 \lambda$. Let $n_{0}$ be the number of central points in modified SOSRD.

Theorem (3.1): Let $\left(v, b, r, k_{1}, k_{2}, b_{1}, b_{2}, \lambda\right)$ be a SUBA with two unequal block sizes,

Case (i): If $r<5 \lambda$, the design points,

$\left[l-\left(v, b, r, k_{1}, k_{2}, b, b, b_{2}, \lambda\right)\right] 2^{2(k)} U n_{0}(a, 0, \ldots ., 0) 2^{1} \cup\left(n_{0}\right)$ give a $v$-dimensional

Modified SOSRD in $N=\frac{\left(r 2^{t(k)}+2 n_{n_{a}} a^{2}\right)^{2}}{\lambda 2^{(k)}}$ design points, if,

$$
\begin{aligned}
& a^{4}=\frac{(5 \lambda-r) 2^{2(k)-1}}{n_{a}}, \\
& n_{0}=\frac{\left(r 2^{\prime(k)}+2 n_{o} a^{2}\right)^{2}}{\lambda 2^{\prime(k)}}-\left[b 2^{\prime(k)}+2 n_{a} v\right]
\end{aligned}
$$

and $n_{0}$ turns out to be an integer.

Case (ii): If $r=5 \lambda$, then the design points,

$\left[1-\left(v, b, r, k_{1}, k_{2}, b_{1}, b_{2}, \lambda\right)\right] 2^{(t)} \cup n_{0}$ give a three level $v$-dimensional modified SOSRD in $N=\frac{\left(r 2^{t(k)}\right)^{2}}{\lambda 2^{f(k)}}$ design points if,

$n_{0}=\frac{\left(r 2^{f(k)}\right)^{2}}{\lambda 2^{\prime(k)}}-b 2^{\prime(k)}$ and $n_{0}$ furns out to be an integer.

Case (iii): If $r>5 \lambda$, then the design points,

$\left[1-\left(v, b, r, k_{1}, k_{2}, b, b_{2}, \lambda\right)\right] 2^{\prime(t)} \cup n_{a}(a, a, a, \ldots, a) 2^{(v)} U n_{0}$ give a $v$-dimensional modified SOSRD in $N=\frac{\left(r 2^{\prime(k)}+n_{\mathrm{o}} 2^{\prime(v)} a^{2}\right)^{2}}{\lambda 2^{(k)}+n_{\mathrm{o}} 2^{(v)} \mathrm{a}^{4}}$ design points if,

$$
a^{4}=\frac{(r-5 \lambda) 2^{t(k)-t(v)-2}}{n_{0}}
$$




$$
n_{0}=\frac{\left(r 2^{t(k)}+n_{0} 2^{\prime(v)} a^{2}\right)^{2}}{\lambda 2^{1(k)}+n_{0} 2^{1(v)} a^{4}}-\left(b 2^{t(k)}+n_{0} 2^{\prime(v)}\right)
$$

and $n_{0}$ furns out to be an integer.

Proof: Case (i): Let $r<5 \lambda$, from conditions of modified SOSRD, we have

$$
\begin{aligned}
& \sum x_{i u}^{2}=r 2^{\prime(k)}+2 n_{a} a^{2}=N \lambda_{2} \\
& \sum x_{i u}^{4}=r 2^{f(k)}+2 n_{0} a^{4}=5 N \lambda_{4} \\
& \sum x_{i u}^{2} x_{i u}^{2}=\lambda 2^{\prime(k)}=N \lambda_{4} .
\end{aligned}
$$

The modified condition $\left(\sum x_{i u}^{2}\right)^{2}=N \sum x_{i v}^{2} x_{i v}^{2}$ leads to $N$ given in equation (3.1). (Alternatively $N$ may be obtained directly as $N=b 2^{\text {t(x) }}+2 v n_{0}+n_{0^{\prime}}$, where $n_{0}$ is is given in equation (3.3)). Equations (3.10) and (3.11) leads to $a^{4}$ given in
equation (3.2).

Case (ii): Lef $r=5 \lambda$, from conditions of modified SOSRD, we have,

$$
\begin{aligned}
& \sum x_{i v}^{2}=r 2^{t(k)}=N \lambda_{2} \\
& \sum x_{i v}^{4}=r 2^{\prime(k)}=5 N \lambda_{4} \\
& \sum x_{i v}^{2} x_{i v}^{2}=\lambda 2^{\prime(k)}=N \lambda_{4}
\end{aligned}
$$

The modified condition $\left(\sum x_{i v}^{2}\right)^{2}=N \sum x_{i v}^{2} x_{i v}^{2}$, leads to $N$ given in equation (3.4). (Alternatively $N$ may be obtained directly as $N=b 2^{\text {t(k) }}+n_{0}$, where $n_{0}$ is given in equation (3.5)). Equations (3.13) and (3.14), we have $r 2^{(t)}=5 \lambda 2^{t(k)}$ implies $r=5 \lambda$.

Case (iii): Let $r>5 \lambda$, from conditions of modified SOSRD, we have,

$$
\begin{aligned}
& \sum x_{i v}^{2}=r 2^{\prime(k)}+n_{a} 2^{\prime(v)} a^{2}=N \lambda_{2} \\
& \sum x_{i v}^{4}=r 2^{t(k)}+n_{a} 2^{\prime(v)} a^{4}=5 N \lambda_{4} \\
& \sum x_{i u}^{2} x_{i v}^{2}=\lambda 2^{\prime(k)}+n_{a} 2^{\prime(v)} a^{4}=N \lambda_{4}
\end{aligned}
$$


The modified condition $\left(\sum x_{i v}^{2}\right)^{2}=N \sum x_{i v}^{2} x_{i v}^{2}$, leads to $N$ given in equation (3.6). (Alternatively $N$ may be obtained directly as $N=b 2^{f(k)}+n a 2^{2(v)}+n_{0}$, where $n_{0}$ is given in equation (3.8)). Equations (3.16) and (3.17), leads to $a^{4}$ given in equation (3.7).

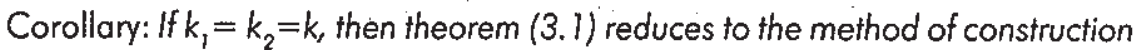
of modified SOSRD using BIBD.

Example (i): Here we construct a modified SOSRD for $v=6$ factors with the help of a SUBA with two unequal block sizes $\left(v=6, b=7, r=3, k_{1}=2, k_{2}=3, b_{1}=3\right.$, $\left.b_{2}=4, \lambda=1\right)$. The design points,

$[1-(6,7,3,2,3,3,4,1)] 2^{3} \cup n_{a}(a, 0, \ldots . ., 0) 2^{1} \cup\left(n_{0}\right)$. Let us fix $n_{a}=2$ Here $r<5 \lambda$. Equations $(3.2) \Rightarrow a^{2}=2.00,(3.1) \Rightarrow N=128$ and $(3.3) \Rightarrow n_{0}=48$. Thus with $c=5, a^{2}=2.00, n_{0}=48$, we get a 6-factor modified SOSRD in $N=128$ design points.

Example (ii): Consider the SUBA with two unequal block sizes, $[1-(v=9, b=18$, $\left.\left.r=5, k_{1}=2, k_{2}=3, b_{1}=9, b_{2}=9, \lambda=1\right)\right] 2^{3}\left(n_{0}\right)$. Here $r=5 \lambda$. Equations (3.5) $\Rightarrow n_{0}=56,(3.4) \Rightarrow N=200$. Thus with $c=5, n_{0}=56$, we get a 9-factor three level modified SOSRD in $N=200$ design points.

\section{Acknowledgements}

The author is thankful to the authorities of Acharya Nagariuna University for providing the necessary facilities to carry out this work

\section{References}

1. Box, G.E.P. and Hunter, J.S. (1957), Multifactor experimental designs for exploring response surfaces, Annals of Mathematical Statistics, 28, 195-241.

2. Das, M.N. and Narasimham, V.L. (1962), Construction of rotatable designs through balanced incomplete block designs, Annals of Mathematical Statistics, 33, 1421-1439.

3. Das, M.N., Rajendra Prasad, and Manocha, V.P. (1999). Response surface designs, symmetrical and asymmetrical, rotatable and modified, Statistics and Applications, 1, 17-34.

4. Hader, R.J. and Park, S.H. (1978). Slope rotatable central composite designs, Technometrics, $20,413.417$. 
5. Jang, D.H. and Park, S.H. (1993). A measure and graphical method for evoluating slope rotatobility in response surface designs, Communications in Statistics - Theory and Methods,
$22,1849-1863$.

6. Park, S.H. (1987). A class of multifactor designs for estimating the slope of response surfaces, Technometrics, 29, 449-453.

7. Raghavarao, D. (1962), Symmetrical unequal block arrangements with two unequal block sizes, Annols of Mathematical Statistics, 33, 620.633.

8. Raghovarao, D. (1971). Construction and Combinatorial Problems in Design of Experiments, John Wiley, New York,

9. Victorbabu, B. Re. (2002a). A note on the construction of four and six level second order slope rotatable designs, Statistical Methods, 4, 11-20.

10. Victorbabu, B. Re. (2002b). Construction of second order slope rotatable designs using symmetrical unequal block arrangements with two unequal block sizes, Journal of the Korean Statistical Society, 31, 153.161.

11. Victorbabu, B. Re, (2003). On second order slope rotatable designs using incomplete block designs, Journal of the Kerala Statistical Association, Vol. 14, 19.25.

12. Victorbabu, B. Re. (2005). Modified second order slope rotatable designs using paimise balanced designs, Proceedings of Andhra Pradesh Akademi of Sciences, Vol. 9, No. 1, 19-22.

13. Victorbabu, B. Re. and Narasimham, V.L. (1991). Construction of second order slope rotatable designs through balanced incomplete block designs, Communications in Statistics -Theory and Methods, 20, 2467-2478. 